\title{
MODELING AND SIMULATING SUPPLY CHAIN SCHEDULE RISK
}

\author{
Gandolf R. Finke \\ BWI Center for Industrial Management \\ ETH Zurich \\ 8032 Zurich, SWITZERLAND
}

\author{
Amanda J. Schmitt \\ Mahender Singh \\ Center for Transportation and Logistics \\ Massachusetts Institute of Technology \\ Cambridge, MA 02142, USA
}

\begin{abstract}
We investigate an aerospace supply chain that is subject to various types of risks in this research. Discrete-event simulation technique is used to model the flow of product and risk factors such as potential supply chain disruptions or quality issues. The underlying goal of the model is to analyze the supply chain performance under various risk scenarios and gather insights. The validity and practical relevance of the results is emphasized as the company is using the model not only for planning, but also for execution and general project management.
\end{abstract}

\section{INTRODUCTION}

In this research we explore risk management issues at an aerospace company, hereafter called Company A. In general, the aerospace industry is unique because of its very low production volumes, which lead to a project management approach to production. It is typical for each unit to take years to complete. Due to the low production volumes and unique nature of the product, inventory buffers are not usually available, making the system highly vulnerable to any potential risk. On time delivery is a commonly used performance metric in the aerospace industry. To this end, we study the impact of different potential disruptions or delay events in the supply chain on the time to complete components or the overall product in this research.

Simulation is a common tool to analyze supply chain risk. Snyder and Shen (2006) analyze the differences between demand and supply uncertainty using simulation models and discuss strategies for coping with each. Deleris and Erhun (2005) develop a Monte Carlo based simulation tool to assess uncertainty in supply networks. Schmitt and Singh (2009a, 2009b) investigate multi-echelon supply chain disruption risk using a combination of Monte Carlo and discrete-event simulation and study the dynamic nature of risk in supply networks. Similarly, we developed a simulation model for the supply chain of Company A that includes various types of disruption/delay possibilities in the product flow. Using this model, we tested several scenarios by adjusting different parameters of the simulation model, some of which are included in this paper,

Our research, however, differs from the available publications in the supply chain risk literature as it does not address the traditional manufacturing regime of batch, mass or flow production to make multiple units. We consider a production system that has an extremely low throughput and takes years to produce a single unit. The model therefore offers insights to certain industries with a similar production setting, such as construction companies or industries engaging in long-term projects. Since the simulation model is actually targeting a schedule risk issue rather than quantity targets and product availability, this approach can also be applied to project management in general, for example product development projects. This research is thus classified in the field of scheduling risk. 


\section{Finke, Schmitt and Singh}

A review of production scheduling theory, classification and practice is given in Graves (1981). There is a significant amount of literature available that is focused on different scheduling problems and algorithms (e.g. Isidore, Back, and Fry 2001; Daniels and Kouvelis 1995). Mulholland and Christian (1999) discuss schedule risk with regards to the construction business and propose a risk assessment process. In this paper we propose a simulation driven solution to assess schedule risk for a supply chain, a methodology which has so far been used mostly for batch/mass/flow production.

The rest of the paper is organized as follows. In Section 2, we detail the background of the project, and in Section 3, we introduce the simulation model. Two different scenarios are described in Section 4, along with a discussion on the implementation of the model in the company. Conclusions are provided in Section 5.

\section{BACKGROUND}

\subsection{Research goal and approach}

Company A initiated this research project with the objective of assessing their risk exposure. We began the investigation by investigating the supply chain and production context of Company A. A Value Stream Map of the critical path of production was developed in collaboration with Company A and served as the structural basis for developing a simulation specifications document. This document describes in detail the supply chain architecture and provides guidelines for model development and analysis. Further, to fulfill research goals, we developed different scenarios together with Company A.

One unique aspect of the model is that there is only one variable that monitors the system performance: time. Time to completion is the sole key indicator of supply chain performance for Company A. The model simulates the production time in order to understand the implications that stochastic influences have on this variable. The product is manufactured one at a time and production of each unit takes a very long time and is assumed to be independent of other units. Once model development and analysis was completed, the model was given to the company for further review and application.

\subsection{System}

\subsubsection{Architecture}

Company A's supply chain consists of a number of production stages with each involving several production sub-processes, quality tests and required material input. Many of these stages and steps are completed by key suppliers or contract manufacturers and those subassemblies are sent to Company A. As mentioned earlier, we focused primarily on the critical production path. While they were not explicitly detailed, the model does check to ensure that non-critical path items are available, meaning that scenarios including disruptions to those items can still be tested. This allowed us to reduce the complexity of the system being modeled while still allowing the most significant risks to be captured and maintaining the quality of results.

A high-level view of the different production stages for the modeled supply chain is shown in Figure 1. Horizontal green boxes represent product stages while vertical yellow boxes represent process steps or tests.

\subsubsection{Risk Factors}

We identified different factors that affect the completion time for components and therefore the overall production duration. These factors were differentiated into three categories based on stochasticity, quality test failures, and disruptions. 


\section{Finke, Schmitt and Singh}

The first category is the stochasticity of production processes. The duration of each production process is rarely deterministic, especially when manual labor and little automation is used. The variability of production processes is therefore one of the key elements contributing to uncertainty in completion time and thus the company's overall risk exposure.

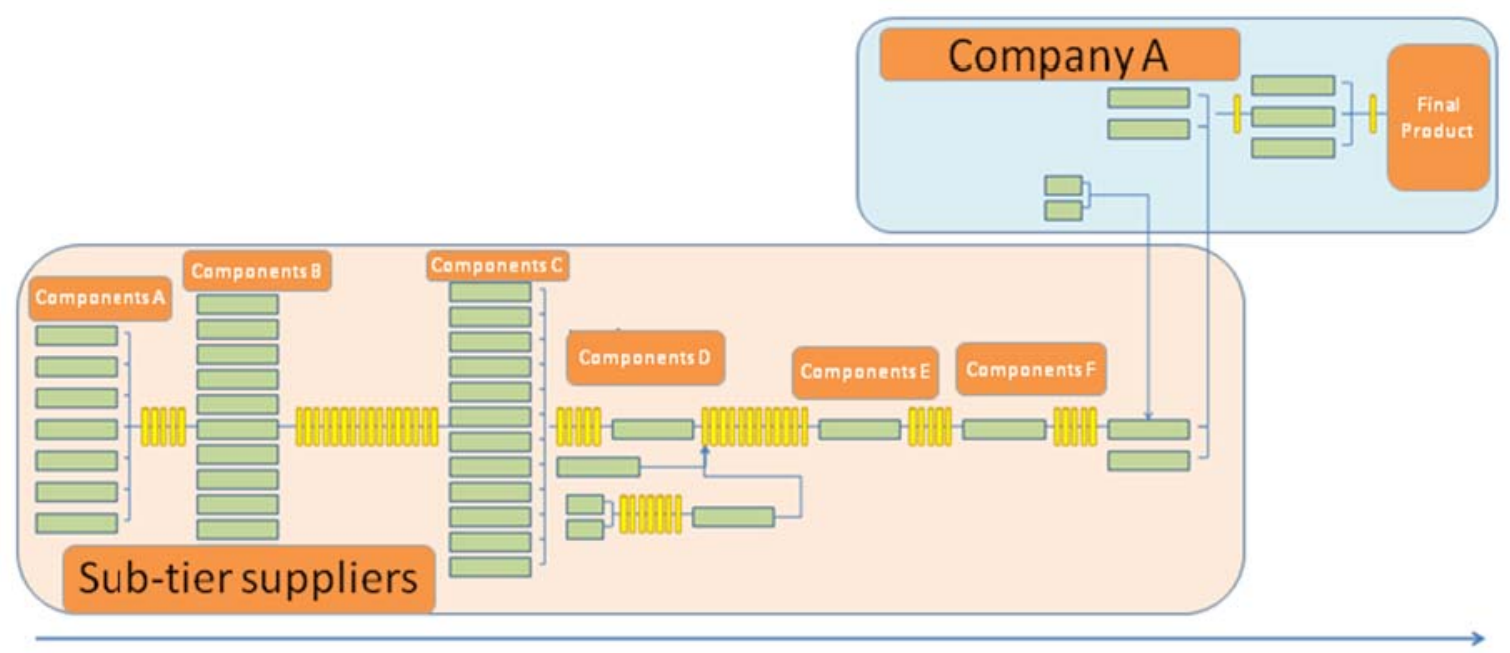

Time

Figure 1: Flowchart of production critical path

The second category is characterized by the possibility of quality test failures. This will drive different outcomes in the product flow depending on which test fails. In one case, a fixed rework time is incurred and/or the preceding step(s) to the quality test must be repeated before the test is conducted again. In the other case, the product needs to be disassembled and rework on preceding components is necessary, so entire segments of the production process must be repeated.

Lastly, supply chain disruptions also affect performance. In this project, supply chain disruptions were split into two types: the availability of necessary parts and the potential disruption of production processes (essentially non-availability of production resources). The availability of necessary parts describes whether parts, either purchased or produced, that are not part of the critical path are available so that production can continue. Hereafter we refer to those parts as "external," as in external to the critical path. If parts are not available, the production is put on hold until the required part becomes available. The second type of disruption, the potential disruption of a production process, covers all incidents that prevent a particular process from completing. The differentiating factor between the two disruption types is that the first type delays the start of a particular production step, while the latter type delays the flow of product. Potential causes for disruptions are manifold and include supplier bankruptcy, quality issues, fires, labor strikes, etc.

\section{SIMULATION MODEL}

This section will introduce the simulation model in more detail and explain how the previously mentioned aspects were implemented.

\subsection{Model Structure and Logic}

The simulation model was created with the simulation software Arena by Rockwell Automation Technologies Inc. based on the specifications described in Section 2. It consists of three different modules: the base model, the disruptions and the interface with Microsoft Excel. We started the modeling 


\section{Finke, Schmitt and Singh}

effort with the base model, which includes all aspects of production except the disruptions, and then added disruptions and integration with Excel.

Figure 2 is a screenshot of the complete simulation model. The base model is shown in a blue frame in the center of the picture, the disruption model is highlighted by the orange and green boxes and the interface functions are highlighted by the red frame.

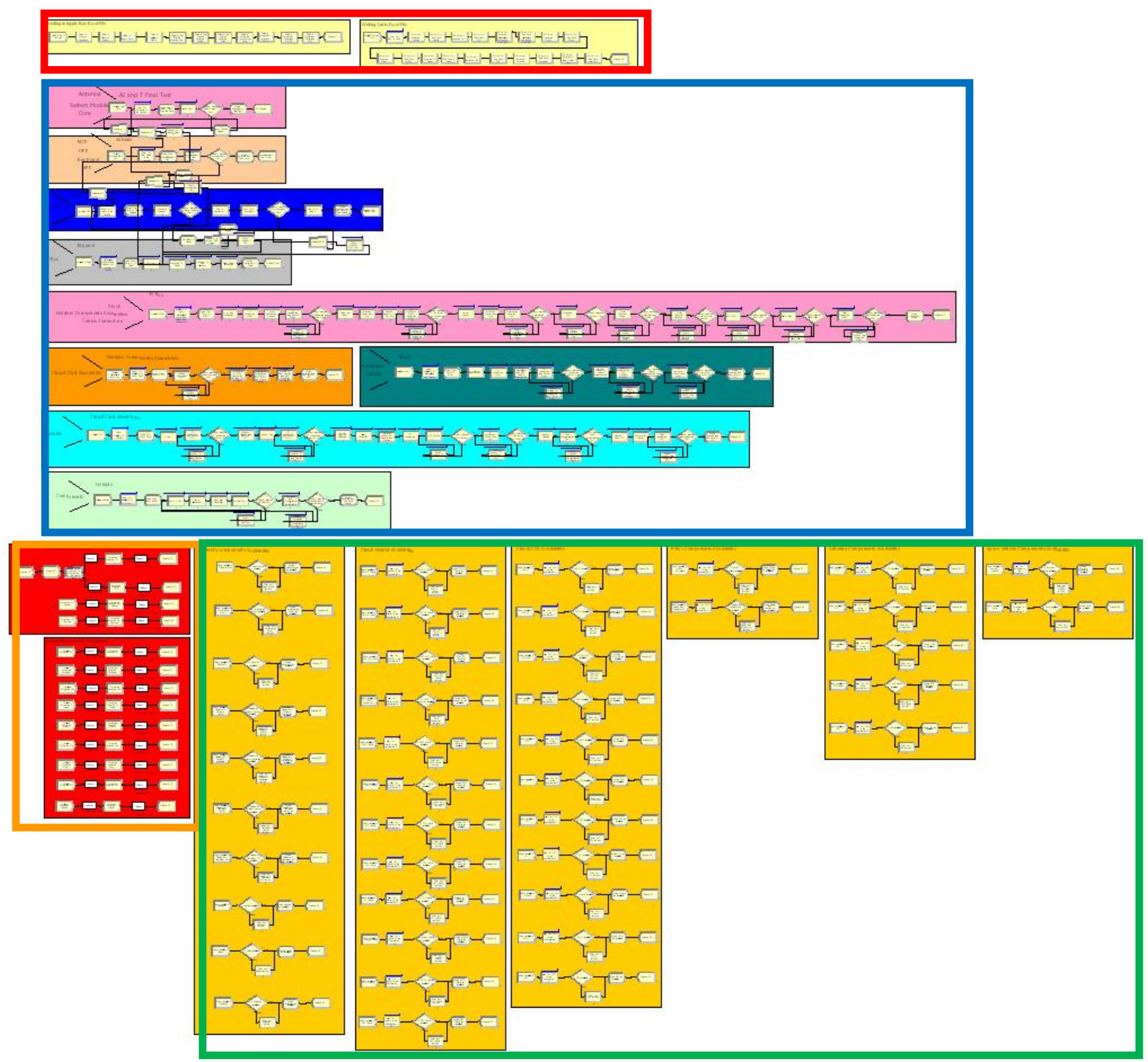

Figure 2: The simulation model including base model, supply chain disruption and interface functions

\subsubsection{Base Model}

The base model is indicated with a blue frame in Figure 2 and contains all production processes in the model. The differently colored boxes within the frame highlight the production of different components within the critical path. Production is initiated for all components at time zero of the simulation. However, all components are prevented from actually being produced until all required sub-components are available, which includes the completion of the preceding component in the critical path.

Every production process is modeled as a delay with a duration that is set according to user-defined parameters. The two parameters are the mean value of the individual process duration and the 


\section{Finke, Schmitt and Singh}

"stochasticity factor." Company A had little information on the nature of the variability of its processes, so based on our discussions with the team we decided to assume that all processes are normally distributed with a mean equal to their deterministic estimate and a standard deviation equal to the mean multiplied by the stochasticity factor. Thus the stochasticity factor is the coefficient of variation for each process. This factor is globally defined for the whole simulation model, which allows for sensitivity testing of system-wide process variability.

The base model also incorporates the potential failures of quality tests. Each test can be regarded as a process. However, there is a user-defined probability of failure. This probability defines the frequency with which product flow continues as planned or rework/disassembly is necessary. The duration of the rework time is variable using the aforementioned stochasticity factor and the logic for what happens after a failure is modeled according to the information provided by Company A. Once the production of one component is successfully completed it is marked as available and the subsequent production sequence can start the production of the next component.

\subsubsection{Supply Chain Disruptions}

After the base model was complete, logic allowing for supply chain disruptions to occur was added. The input parameters for the delay of a component are the probability of occurrence and the duration, or severity, defined using three parameters of a triangular distribution. The parameters of a triangular distribution are the minimum, the maximum and the most likely value of delay. We chose to use a triangular distribution because its inputs are straightforward and easy for Company A members to understand and manipulate in the future. The occurrence and severity of disruptions can be set to appear randomly according to a probability distribution or deterministically. The model can therefore be both deterministic and stochastic, depending on the inputs. This is particularly valuable when testing specific scenarios where parts of the model are set to be deterministic in order to isolate the stochasticity.

The other disruption risk consists of a sudden non-availability of production resources. For every production process we distinguish between a potential micro and macro disruption. The micro disruption only affects a production sequence and is therefore focused on specific production processes. The macro disruption affects a whole location and seizes all resources at that particular site regardless of the production sequence that is currently in progress.

In this model, we included two different resource types that can be disrupted independently of each other. One is component-based and the other location-based. Component-based disruptions affect any process within a specific production sequence, e.g. the malfunction of machinery that is required for carrying out a specific production sequence. Location-based disruptions affect all production sequences that have been grouped to be in the same location. One example would be a hurricane or a fire at a production site. Both the component- and the location-based disruptions are characterized through two factors: the inter-arrival time (we assume a Poisson process since these events are independent and memoryless) and the duration until production can continue (again assuming a triangular distribution).

\subsubsection{Interface - Input and Output}

What we refer to as the interface of the model is also necessary in order for the model to run and collect data. The model has an interface with Excel such that all input parameters are read in from an Excel sheet and relevant results are written out to an Excel sheet as well. The random values themselves are not read in, but the parameters that define the probability distributions are. The random values are created within the Arena model.

The interface with Excel was created to enhance the ease of use and thus increase acceptance at Company A. Additionally, not only does this add to the user-friendliness, it also provides data in a format ready to be processed further. 


\section{Finke, Schmitt and Singh}

As explained earlier, time is the only relevant measurement unit. The output provided by the model contains the duration and finishing time of each production sequence, with the last sequence's finishing time being the overall completion time of the product. We ran 1,000 replications for each simulation run, which proved to be sufficient for analysis of the stochastic performance of the system.

\subsection{Model Verification and Validation}

Model verification serves the purpose of analyzing whether the model works as intended, while validation considers aspects of how relevant the simulation model is with regards to the system in reality (Law 2003). In addition to collaborating regularly with members of Company A, more measures were implemented to verify and validate the simulation model.

We conducted a truly deterministic run of the simulation disabling all stochastic influences. As expected, the results of the simulation runs were constant and we verified that the completion time equaled the sum of all appropriate input process durations. Visual evaluation of the animation during simulation runs added to verification as well. We also experimented by adjusting the value of single parameters and observed whether performance changed in the predicted direction and to an appropriate extent. In addition to reviewing the model flow with Company A, a key supplier of Company A also reviewed the model and its outputs and provided insights and validation.

\section{SCENARIOS AND OUTCOME}

Several scenarios were developed according to the risk sources identified as part of this project. The specific scenarios run were determined in collaboration with Company A. Aside from scenarios focused on disruptions, we also ran a baseline scenario in which all inputs were set equal to the most realistic input values. This baseline scenario served as a reference when analyzing the influence of specific parameters.

In this section, two scenarios are presented which consider different possible situations or configurations and their effect on completion time. We show how the simulation model can be applied in practice by analyzing the supply chain performance sensitivity to changes in parameters.

\subsection{The Stochasticity Factor}

The stochasticity factor influences the variability of all production durations in the model. In this scenario, we focused on how process variability affects the overall supply chain performance.

We include five different parameter values for the stochasticity factor: $0 \%, 20 \%, 27 \%$ (the baseline scenario), $40 \%$ and $100 \% .0 \%$ describes the deterministic scenario in terms of duration variability, but stochastic failure rates for quality tests were still included. A $100 \%$ stochasticity factor sets the standard deviation of every process duration to be the same value as its mean. The $27 \%$ stochasticity factor is deemed to be the most realistic representation of the Company A situation and hence called the baseline scenario. This stochasticity value was arrived at after a detailed discussion and investigation in close cooperation with Company A and a key supplier. It is generated by calculating the weighted average of the perceived stochasticity of various process durations of different components. Validation of this stochasticity factor came from sharing the output of the simulation again with Company A and the key supplier for confirmation.

Figure 3 shows the impact of the stochasticity factor on completion time of the product. The graph ranks the completion time for 1,000 simulation runs in percentiles. The percentiles also serve as the estimates of the confidence level of finishing the product in a given time.

Mathematically, each curve in Figure 3 should have the same value at the 50th percentile point because a Normal distribution ranges from negative infinity to positive infinity. However, the numbers do not exactly match since we cannot let the realization for processing times go negative (whereas true, nontruncated normal distributions do allow for negative realizations). Instead, our lowest value is 0 and our 


\section{Finke, Schmitt and Singh}

highest limit is positive infinity. Thus, as stochasticity rises, so does the value at the 50th percentile point (or median). The graph illustrates how important it is to set the stochasticity factor to the correct value.

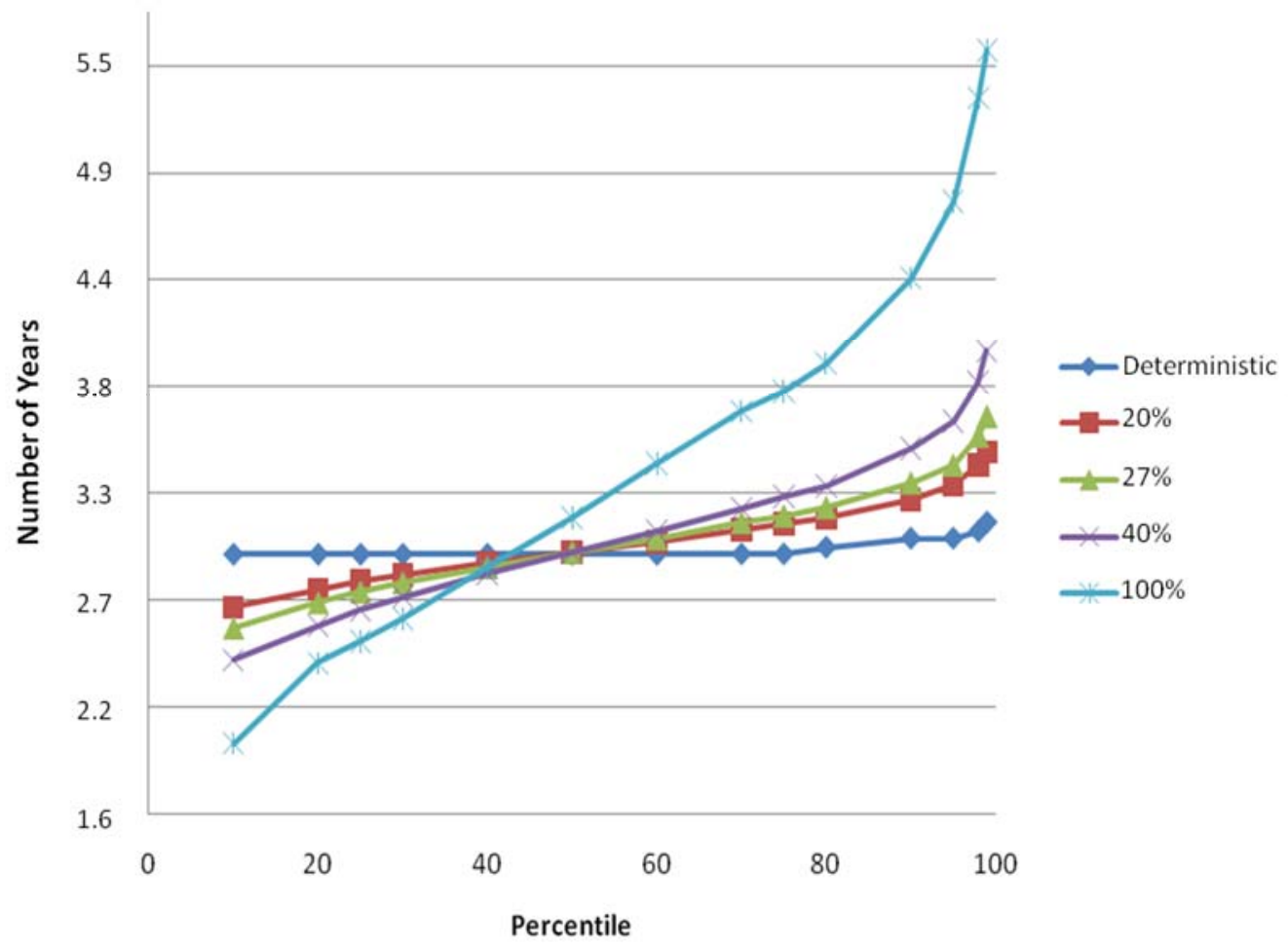

Figure 3: Percentiles for different levels of variability

Table 1 provides the actual numeric values in days shown in Figure 3. For confidence levels greater than $50 \%$, the increasing gap between the different levels of variability is obvious.

Table 1: Numeric values for different stochasticity factors (in terms of total duration in days)

\begin{tabular}{|c|c|c|c|c|c|}
\hline Percentile & Deterministic & $\mathbf{2 0} \%$ & $\mathbf{2 7} \%$ & $\mathbf{4 0} \%$ & $\mathbf{1 0 0} \%$ \\
\hline $10 \%$ & 1085 & 987 & 948 & 888 & 732 \\
\hline $20 \%$ & 1085 & 1020 & 996 & 952 & 882 \\
\hline $25 \%$ & 1085 & 1035 & 1016 & 983 & 922 \\
\hline $30 \%$ & 1085 & 1047 & 1033 & 1006 & 966 \\
\hline $40 \%$ & 1085 & 1069 & 1062 & 1050 & 1062 \\
\hline $50 \%$ & 1085 & 1090 & 1090 & 1089 & 1154 \\
\hline $60 \%$ & 1085 & 1110 & 1116 & 1129 & 1256 \\
\hline $70 \%$ & 1086 & 1131 & 1146 & 1171 & 1353 \\
\hline $75 \%$ & 1086 & 1143 & 1158 & 1193 & 1391 \\
\hline $80 \%$ & 1098 & 1153 & 1174 & 1213 & 1443 \\
\hline $90 \%$ & 1115 & 1187 & 1220 & 1283 & 1604 \\
\hline $95 \%$ & 1115 & 1215 & 1253 & 1335 & 1747 \\
\hline $98 \%$ & 1128 & 1253 & 1307 & 1408 & 1940 \\
\hline $99 \%$ & 1145 & 1277 & 1344 & 1466 & 2029 \\
\hline
\end{tabular}




\section{Finke, Schmitt and Singh}

These numbers are also useful in determining the extent of deviation from the expected completion time, even when no disruptions are present. This can have an impact on strategic decision-making. For example, using these values for a given completion date, Company A can derive the expected penalty costs and compare this to the required investment to lower the variability to increase the confidence in the promised completion date. Investments in lowering variability could include increasing automation or incentivizing workers. Alternately, Company A can use these values to set their completion dates with a given confidence level.

\subsection{Disruptions on the Component Level}

In this scenario the sensitivity of the overall supply chain performance is tested with respect to disruptions of different durations. We choose a specific supplier, set the probability of delay to $100 \%$ and set the delay itself equal to 20,60 or 120 days based on input from Company A. In Figure 4 we can clearly see that the disruption deterministically increases the completion time by 20,60 or 120 days respectively compared to the baseline scenario. This upward shift means that the original target date can now be reevaluated with a new confidence level for whether it will be met. For example, if the original committed target date was 3.1 years, which provides a confidence level of $60 \%$ under the baseline scenario, and if a 120-day disruption were to occur, this confidence level drops to below $20 \%$. This information output by the simulation can thus be used to evaluate the current risk of missing the scheduled target date and management can react accordingly.

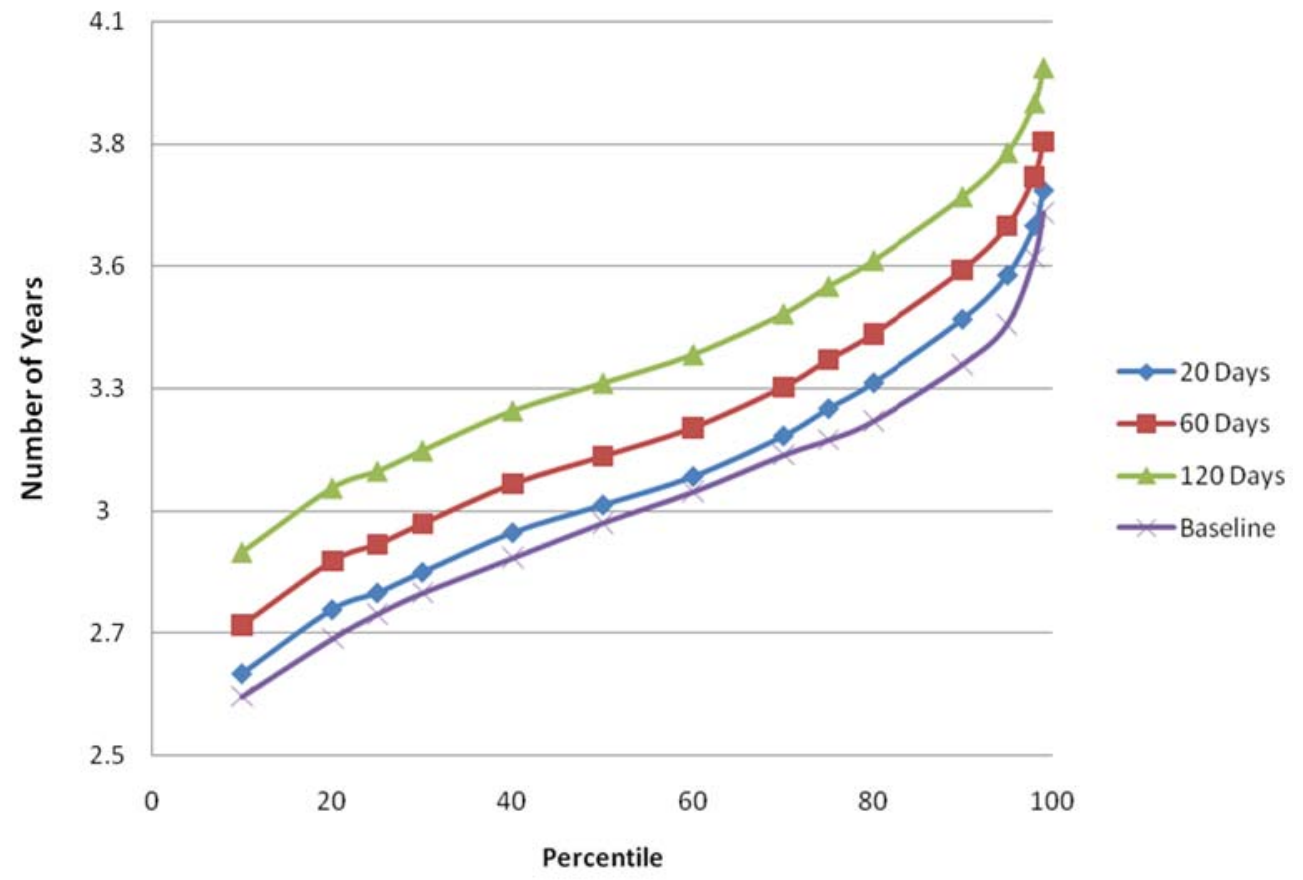

Figure 4: Component-level disruption for 20, 60 and 120 days

We ran simulations with disruptions at different stages of production and observed this effect every time. A key real life implication from this scenario is that a delay early on in the project is potentially less harmful because there is more opportunity to make up the time lost, and thus could potentially be addressed better than a disruption in the final phase. However, corresponding human reactions to disruptions might have an influence on overall completion time that are not captured with this simulation model. 


\section{Finke, Schmitt and Singh}

An example of how this analysis could have an impact is to consider how the delay penalties for suppliers should be structured. A threshold value, above which a delay causes an unacceptable drop in performance, could be determined using this model and plans and fees could be put in place to cope with such a delay.

\section{CONCLUSIONS}

We have shown how the underlying issue of completion time analysis of a one-product supply chain can be simulated including several stochastic risk influences. The background of the simulation project was outlined and we introduced the basic logic of the simulation model. We presented a description of different stochastic influences affecting the supply chain performance and how we implemented the same. Two different scenarios that were tested with the simulation model were discussed to offer insights to decision-makers.

A key outcome of the component-level disruption analysis discussed in Section 4.2 is that small suppliers, which Company A has little control over, play an important role in the risk levels. Analysis showed that disruptions at any stage and from any supplier can have severe impact on the supply chain performance. These results justify a high level of scrutiny of even a small supplier from a risk management perspective.

The model intentionally contains only a single measurement unit: time. This adds to the comprehensibility and ease of use of this model, which has increased its acceptance at Company A. The simulation model provides a basis to analyze the stochasticity of overall completion times. However, the simulation model also includes a number of assumptions and simplifications such as using the same stochasticity factor for production process durations. If this assumption were to be regarded as too limiting, the model could easily be adapted appropriately by introducing more stochasticity factor variables. A single stochasticity factor, however, seems reasonable as long as there is not sufficient data indicating large differences between production processes.

The results of this study have influenced the way that Company A approaches disruption risk management in general. We showed that one very important factor in determining the impact of a given disruption is the duration that production is actually halted, which depends on recovery strategy and speed. Thus Company A is shifting their general risk management approach to focus more on recovery speed and general resilience than on specific risk source identification.

A demonstration of the value of this research is the fact that the simulation model is being actively used by Company A. Further efforts are being made to refine the model input and new scenarios are also being considered for evaluation to gain more insights. Model output is being used to determine the current confidence level in finishing their first production run on time and to evaluate specific risks in their system. Interestingly, Company A is not only using the model as a planning tool to identify critical supply chain vulnerabilities, but also intends to use the model in real time to assess the current schedule risk in the supply chain dynamically and update the predicted product completion time. This application is facilitated by the ability of the model to capture the complete state of the system at any given time using a single variable, time, which is unambiguously known to the decision-maker.

\section{ACKNOWLEDGEMENTS}

The authors would like to express their thanks to Florian Naegele, a visiting research student at the Center for Transportation and Logistics at MIT at the time this project was carried out. His work in risk identification and related issues significantly contributed to the success of this project. We also thank Bryan Keith Wilson, a student in the 2010 class of MIT's Master of Engineering in Logistics program, for valuable help with creating and analyzing scenarios, data and figures. Finally, our thanks go to the team members from Company A, who sponsored this work; their input was necessary, valuable and much appreciated by all team members. 
Finke, Schmitt and Singh

\section{REFERENCES}

Daniels, R.L., and P. Kouvelis. 1995. Robust scheduling to hedge against processing time uncertainty in single-stage production. Management Science 41(2):363-376.

Deleris, L. A., and F. Erhun. 2005. Risk management in supply networks using monte-carlo simulation. In Proceedings of the 2005 Winter Simulation Conference, ed. M. E. Kuhl, N. M. Steiger, F. B. Armstrong and J. A. Joines, 1643-1649. Piscataway, New Jersey: Institute of Electrical and Electronics Engineers, Inc.

Graves, S. C. 1981. A Review of Production Scheduling. Operations Research 29 (4):646-675.

Isidore, L.J., W.E. Back, and G.T. Fry. 2001. Integrated probabilistic schedules and estimates from project simulated data. Construction Management \& Economics 19(4):417-426.

Law, A. 2003. How to conduct a successful simulation study. In Proceedings of the 2003 Winter Simulation Conference, ed. S. Chick, J. Sanchez, D. Ferrin, and D.J. Morrice, 66-70. Piscataway, New Jersey: Institute of Electrical and Electronics Engineers, Inc.

Mulholland, B., and J. Christian. 1999. Risk assessment in construction schedules. Journal of Construction Engineering and Management 125(1):8-15.

Schmitt, A. J., and M. Singh. 2009a. Quantifying supply chain disruption risk using Monte Carlo and discrete-event simulation. In Proceedings of the 2009 Winter Simulation Conference, ed. M. D. Rossetti, R. R. Hill, B. Johansson, A. Dunkin, and R. G. Ingalls, 1237-1248. Piscataway, New Jersey: Institute of Electrical and Electronics Engineers, Inc.

Schmitt, A. J., and M. Singh. 2009b. A quantitative analysis of disruption risk in a multi-echelon supply chain. Working paper, Center for Transportation and Logistics, MIT, Cambridge, MA.

Snyder, L.V., and Z .J. M. Shen. 2006. Supply and demand uncertainty in multi-echelon supply chains. Working paper, P.C. Rossin College of Engineering and Applied Sciences, Lehigh University, Bethlehem, PA.

\section{AUTHOR BIOGRAPHIES}

GANDOLF R. FINKE is a Ph.D. student at the Center for Industrial Management (BWI) at ETH Zurich. His research focuses primarily on quantitative aspects of supply chain risk management with a special interest in simulation. Prior to his current studies he received his Diploma in Industrial Engineering and Management from the University of Karlsruhe (TH) and gained international experience in different industrial and academic institutions including a ten month research stay at the Center for Transportation and Logistics at MIT. His email address is $\langle g$ finkedethz. ch>.

AMANDA J. SCHMITT is a Postdoctoral Associate at the Center for Transportation and Logistics at MIT. Her research focuses on designing and managing supply chains to protect against supply disruption risk. She received her Ph.D. in Industrial Engineering in 2008 from Lehigh University. In September 2010 she will join McKinsey \& Company as an Associate in their Philadelphia office. Her email address is <aschmittemit.edu>.

MAHENDER SINGH is a Research Director at the Center for Transportation and Logistics at MIT. His research focuses on operations and supply chain management, with particular interest in exploring the underlying structure of complex supply chains. He received his Ph.D. from the University of Tennessee, Knoxville, and has over fifteen years of experience in the field of supply chain management. His email address is $<$ msingh@mit.edu>. 